\title{
Évaluation du délai DIDO (door in-door out) des syndromes coronariens aigus dans les hôpitaux sans cardiologie interventionnelle du réseau RESSIF
}

\section{Evaluation of the Door In-Door Out (DIDO) time of acute coronary syndrome in hospitals without coronary care unit of the network RESSIF}

\author{
C. Cahuzac $\cdot$ T. H. Ta $\cdot$ A. Henaoui $\cdot$ F.-X. Laborne $\cdot$ N. Briole $\cdot$ M. Porche $\cdot$ L. Lamhaut $\cdot$ D. Sapir $\cdot$ H. Andrianjafy \\ Reçu le 10 août 2017 ; accepté le 24 janvier 2018 \\ (C) SFMU et Lavoisier SAS 2018
}

Résumé Objectifs : Le délai door in-door out (DIDO) représente la durée de séjour au service d'urgence (SU) des patients pris en charge pour un syndrome coronarien aigu $\mathrm{ST}+(\mathrm{SCAST}+)$, avant transfert en centre de cardiologie

C. Cahuzac $\cdot H$. Andrianjafy $(\bowtie)$

Service des urgences, hôpital de Longjumeau,

groupe hospitalier Nord-Essonne, 159,

rue du Président-François-Mitterrand,

F-91160 Longjumeau, France

e-mail : h.andrianjafy@gh-nord-essonne.fr

T. H. Ta

SMUR 91, hôpital de Longjumeau,

groupe hospitalier Nord-Essonne, 159,

rue du Président-François-Mitterrand,

F-91160 Longjumeau, France

T. H. Ta $\cdot$ D. Sapir $\cdot$ H. Andrianjafy

RESSIF — réseau des urgences Sud Île-de-France

F-91160 Longjumeau, France

A. Henaoui · M. Porche

Service des urgences, hôpital Arpajon,

18, avenue de Verdun, F-91294 Arpajon, France

F.-X. Laborne · D. Sapir

SAMU 91, 40, avenue Serge-Dassault,

F-91100 Corbeil-Essonnes, France

N. Briole

SAMU 77, service des urgences,

centre hospitalier Marc-Jacquet,

2, rue Fréteau-de-Peny, F-77000 Melun, France

L. Lamhaut

Département d'anesthésie-réanimation,

SAMU de Paris, CHU Necker-Enfants malades,

Assistance publique-Hôpitaux de Paris,

149, rue de Sèvres, F-75015 Paris, France

Université Paris-Descartes, Centre d'expertise de la mort subite, UMR INSERM 070, PARCC, F-75016 Paris, France interventionnelle (CCI). Le DIDO ne devrait pas dépasser 30 minutes. Notre objectif était d'évaluer le DIDO dans le réseau des urgences Sud Île-de-France (RESSIF).

Matériel et méthodes : Étude épidémiologique, rétrospective, multicentrique comparant le DIDO entre 2014 et 2015 (période 1), puis en 2016 (période 2) dans deux hôpitaux sans CCI, l'hôpital de Longjumeau et l'hôpital d'Arpajon. En 2016 était appliqué dans notre protocole régional $\mathrm{SCAST}+$ le « réflexe électro-oculotéléphonique » (REOT), avec l'objectif de diminuer le DIDO : dès l'électrocardiogramme qualifiant (ECGq), avant tout traitement ou obtention du CCI de transfert, le médecin urgentiste appelle le service d'aide médicale à l'urgence (SAMU) pour l'envoi immédiat d'un service mobile d'urgence et de réanimation (SMUR) secondaire.

Résultats : Entre les deux périodes, le délai DIDO médian n'a pas été modifié significativement ( -16 min [4-42] ; $p=0,06)$. Le délai entre ECGq et appel du SAMU n'était pas significativement différent avant et après application du REOT (15 [8-34] vs 9 min [6-26] ; $p=0,12$ ). Le DIDO était supérieur ou égal à 30 min en période 1 , contre $6 \%$ en période $2(p=0,11)$.

Conclusion : Le DIDO du RESSIF n'a pas été significativement modifié. Il n'y a pas eu d'impact significatif du REOT sur le DIDO, mais notre étude manque de puissance. Le REOT pourrait être évalué plus largement dans les SU. Un réseau de soins impliquant les SAMU, SMUR, SU et CCI avec protocoles partagés est essentiel pour le pronostic des SCAST+.

Mots clés Syndrome coronarien aigu ST $+\cdot$ Service d'urgence · délai DIDO (door in-door out) $\cdot$ Réseau de soins

Abstract Objectives: The "door in-door out" (DIDO) time is the length of stay in emergency department (ED) for 
ST-elevation myocardial infarction (STEMI) patients, prior transportation to a coronary care unit (CCU). DIDO time should not exceed $30 \mathrm{~min}$. Our objective was to assess the DIDO in the regional network of care RESSIF (RESeau des urgences Sud Ile de France).

Methods: Epidemiological, retrospective, multicenter study comparing the DIDO time between 2014-2015 (period 1) and 2016 (period 2), in two hospitals without CCU, Hospital of Longjumeau and Hospital of Arpajon. In 2016, the « Reflex Electro-Eye-Telephone » (REET) was applied in our regional STEMI protocol, with the goal to reduce the DIDO time: As soon as the electrocardiogram (ECG) was seen, before treatment or getting CCU destination, the emergency physician calls the "service d'aide médicale à l'urgence (SAMU)", to get at the earliest a medical transport "service mobile d'urgence et de réanimation (SMUR) secondaire".

Results: Between the two periods, the DIDO time was not significantly modified $(-16 \min [4-42], p=0.06)$. The ECG-Call to SAMU time was not significantly different before and after applying the REET (15 min [8-34] vs $9 \min [6-26], p=0.12$ ). DIDO time was never $\leq 30 \mathrm{~min}$ in period 1 , while $6 \%$ in period $2(p=0.11)$.

Conclusion: The DIDO time was not significantly modified. There was no significant impact of the REET on the DIDO, but our study is underpowered. The REET could be assessed more widely in EDs. Regional network of care involving SAMU, SMUR, ED, and CCU with shared protocols is a prerequisite for the prognosis of STEMI.

Keywords ST-elevation myocardial infarction - Emergency department $\cdot$ Door in-door out (DIDO) time $\cdot$ Regional network

\section{Introduction}

Le syndrome coronarien aigu avec sus-décalage du segment ST (SCAST+) est une urgence thérapeutique. Le pronostic des patients est directement lié au délai de reperfusion coronaire, et l'angioplastie coronaire percutanée (ACP) est la méthode de reperfusion de référence [1]. En France, $50 \%$ des SCAST+ arrivent spontanément dans les services d'urgence (SU) sans utiliser la filière « idéale » utilisant le service d'aide médicale urgente (SAMU) [2,3]. Cette filière idéale permet un diagnostic, un traitement précoce et une orientation du patient directement en centre de cardiologie interventionnelle (CCI). Quand un patient se présente hors de cette filière, il risque de consulter un hôpital sans CCI et doit être transféré secondairement en CCI.

L'American College of Cardiology (ACC), l'American Heart Association (AHA) et l'European Society of Cardio- logy (ESC) ont défini récemment un nouvel indicateur de performance des SCAST+ pour les hôpitaux sans CCI : le door in-door out (DIDO) [4,5]. Il correspond au délai de séjour du patient SCAST+ au SU avant son transfert en CCI. Le DIDO ne devrait pas dépasser $30 \mathrm{~min}$, ce délai étant corrélé à un risque plus élevé de mortalité. Wang et al. ont constaté un taux de décès de $6 \%$ pour un DIDO supérieur à $30 \mathrm{~min}$, versus $3 \%$ pour un DIDO inférieur ou égal à $30 \mathrm{~min}$ $(p<0,001)$ [6]. Le DIDO est devenu une priorité dans la prise en charge et le pronostic des SCAST + dans les hôpitaux ne disposant pas de CCI.

L'objectif principal de notre étude était d'évaluer le DIDO et son évolution dans le temps dans des SU sans CCI du réseau des urgences Sud Île-de-France (RESSIF). Les objectifs secondaires étaient de mesurer le DIDO et son écart aux recommandations, d'évaluer si l'introduction du réflexe électro-oculotéléphonique (REOT) dans le RESSIF améliorait le DIDO et de mesurer l'impact du DIDO sur le taux de thrombolyse intraveineuse.

\section{Matériel et méthodes}

Il s'agit d'une étude épidémiologique rétrospective et multicentrique. Le RESSIF est un réseau régional d'urgence créé en 2013 sur un modèle préconisé par les recommandations internationales $[4,5]$. La filière de soins du SCA regroupe l'ensemble des SU, SAMU, des services mobiles d'urgences et de réanimation (SMUR), des unités de soins intensifs cardiologiques et des CCI des hôpitaux du département 91 (Essonne) étendu au Sud 77 (Seine-et-Marne). Un protocole commun à toutes ces structures a été rédigé sur le SCAST+, et un recueil permanent de tous les SCAST+ permet de suivre et d'évaluer son application dans le RESSIF (Annexe). Ce recueil est inspiré du registre «e-MUST » (évaluation en médecine d'urgence des stratégies thérapeutiques des SCAST + de moins de 24 heures), relatif à la prise en charge des SCAST+ par les SAMU/SMUR d'Île-de-France [7]. À partir du recueil des SCAST+, nous avons analysé le DIDO au sein du RESSIF durant deux périodes : la période 1 allait du $1^{\text {er }}$ janvier 2014 au 31 décembre 2015 , la période $2 \mathrm{du} 1^{\text {er }}$ janvier au 31 décembre 2016. En début 2016, le REOT a été introduit dans le protocole RESSIF : dès le diagnostic de SCAST+ fait sur l'électrocardiogramme qualifiant (ECGq), avant tout traitement ou obtention de destination finale de transfert du patient, le médecin urgentiste appelle le SAMU, qui fait immédiatement partir un SMUR secondaire pour assurer le transfert en CCI. L'examen clinique, le traitement et le choix du CCI sont effectués secondairement en attendant l'arrivée au SU du transport médicalisé. Puis le médecin régulateur du SAMU est rappelé pour confirmer les modalités de prise en charge (complications cliniques éventuelles, traitements institués). L'objectif 
du REOT est d'obtenir le plus tôt possible le SMUR secondaire. À notre connaissance, il n'y a aucune étude publiée sur ce principe du REOT; notre démarche est donc en ce sens originale.

Notre étude a concerné les SCAST + des SU de deux hôpitaux pilotes du RESSIF, non équipés de cardiologie interventionnelle, le centre hospitalier Longjumeau (CHL) et le centre hospitalier Arpajon (CHA). Le SU du CHL accueille 35000 urgences par an, la permanence de soins de nuit pour les urgences médicales est assurée par un praticien du service et un interne diplômé en études spécialisées (DES) de médecine générale. Il existe au CHL une USIC non interventionnelle de 10 lits avec un cardiologue de garde 24 heures sur 24. Le SU du CHA accueille 30000 patients par an. Un praticien du SU assure la permanence de soins. Un cardiologue assure des consultations certains jours de la semaine, mais il n'y a pas de service de cardiologie. En cas de SCAST+ dans ces deux hôpitaux, les patients devant avoir une ACP sont transférés dans les CCI du département ou plus rarement hors département. Trois CCI existent dans le département 91, permettant le plus souvent de prendre en charge l'ensemble des patients du RESSIF qui nécessitent une ACP : le centre hospitalier sud-francilien de CorbeilEssonnes (CHSF), l'institut cardiovasculaire Paris-Sud de l'hôpital privé Jacques-Cartier de Massy (ICPS), l'hôpital privé Claude-Galien de Quincy-sous-Sénart.

Les critères d'inclusion étaient un âge supérieur à 18 ans, un délai « symptômes-arrivée au SU » inférieur à 24 heures. Le diagnostic de SCAST+ était l'association d'une douleur typique depuis plus de 20 min résistant à la trinitrine, ou des symptômes atypiques coronariens tels que dyspnée, malaise, instabilité hémodynamique, et des anomalies ECG avec un sus-décalage de ST supérieur ou égal à $2 \mathrm{~mm}$ dans deux dérivations contiguës ( $1 \mathrm{~mm}$ d'écart-type) ou un bloc de branche gauche présumé récent. Les patients devaient être transférés en CCI à l'issue de la prise en charge au SU.

Les critères de non-inclusion étaient les patients décédés au SU, les SCA ST+ diagnostiqués dans un service de l'hôpital autre que le $\mathrm{SU}$, les patients en arrêt cardiaque au moment de la prise en charge au SU et avant l'ECGq. Les patients amenés médicalisés par un SMUR n'étaient pas inclus : dans ce cas, le premier contact médical et donc l'ECGq étaient considérés comme le moment de prise en charge extérieure par le médecin du SMUR, ce qui rend difficile l'interprétation du DIDO. En cas de décision médicale au SU de non-transfert en CCI, le patient n'était pas inclus : ce critère ne concerne, dans notre protocole RESSIF, que les patients en limitation de soins pour lesquels un acte invasif paraît déraisonnable.

Le critère de jugement principal était la mesure du DIDO que nous avons déterminée sur les périodes 1 et 2. Le DIDO représente le temps total de passage ou la durée de séjour dans le SU d'un patient SCAST+, avant son transport médicalisé en CCI. Ce délai est lui-même divisé en plusieurs sous-délais qui détaillent les différentes étapes de prise en charge au SU [8,9] (Fig. 1).

Les critères de jugement secondaires étaient l'analyse des sous-délais du DIDO, la proportion de DIDO inférieur au seuil recommandé de 30 minutes et le taux de thrombolyse intraveineuse.

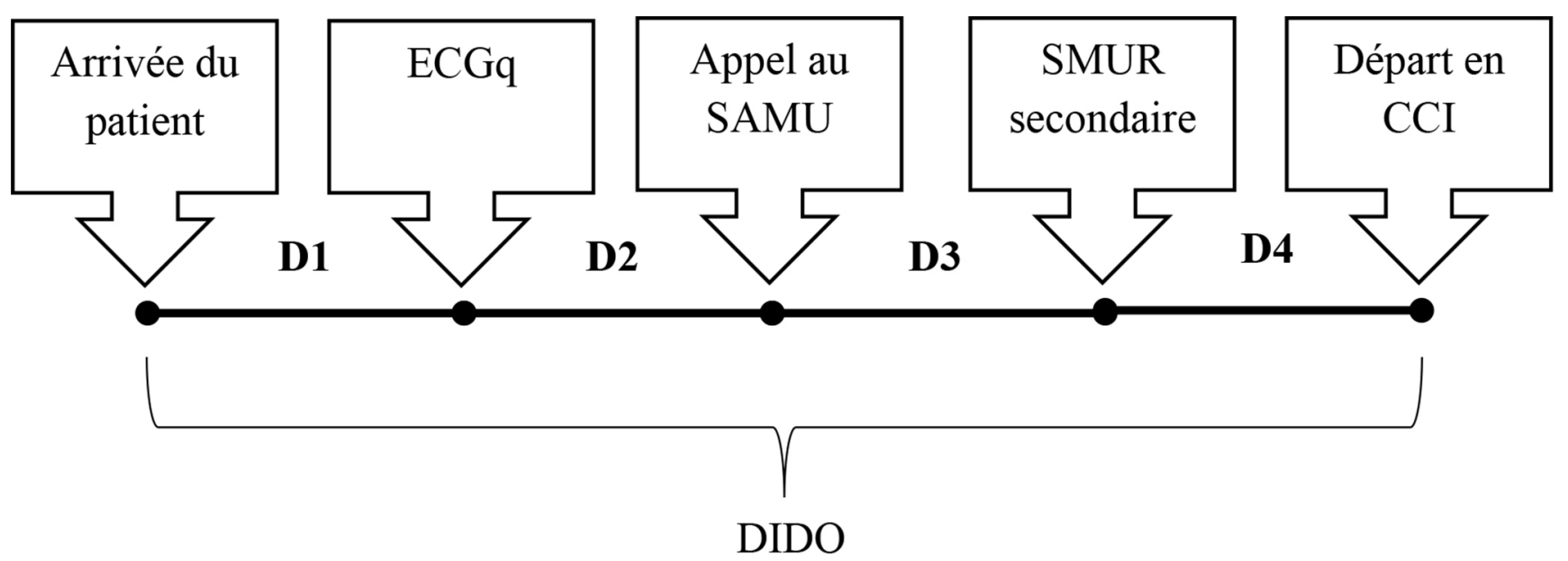

Fig. 1 Définition du délai DIDO (door in-door out) au SU ; D1 : heure d'arrivée du patient au SU $\rightarrow$ heure de l'ECGq ; D2 : heure de l'ECGq $\rightarrow$ heure d'appel au SAMU pour demande de SMUR secondaire ; D3 : heure d'appel au SAMU $\rightarrow$ heure d'arrivée au SU du SMUR secondaire ; D4 : heure d'arrivée SMUR secondaire $\rightarrow$ heure de départ du SU en CCI ; SU : service d'urgence ; ECGq : électrocardiogramme qualifiant ; SAMU : service d'aide médicale à l'urgence ; SMUR : service mobile d'urgence et de réanimation ; CCI : centre de cardiologie interventionnelle 


\section{Analyse statistique}

Les variables quantitatives (délais en minutes) sont exprimées en médiane avec interquartiles et comparées par un test non paramétrique de Mann-Whitney. Les variables catégorielles sont exprimées en pourcentage et comparées par un test $\mathrm{du} \mathrm{Chi}^{2}$ ou un test exact de Fisher selon les effectifs. Tous les tests ont été réalisés de façon bilatérale, avec un risque de première espèce alpha fixé à $5 \%$. Les analyses ont été réalisées à l'aide du logiciel $\mathrm{R}(\mathrm{R}$ version 3.3.1, (C) 2016 The R Foundation for Statistical Computing).

\section{Résultats}

Soixante-huit patients ont été inclus au cours de la période 1 et 34 au cours de la période 2. L'âge moyen des patients était superposable au cours des deux périodes, 63 ans \pm 16 versus 62 ans $\pm 14(p=0,86)$. La proportion de femmes était plus importante au cours de la période 1 , 46 contre $24 \%(p=0,04)$. Le délai médian douleur thoracique-SU était respectivement de 160 [87-249] et $123 \mathrm{~min}$ [68-224] $(p=0,41)$ en période 1 et période 2.

La différence absolue entre les délais médians DIDO des périodes 1 et 2 était de $16 \min$ [4-42] $(p=0,06)$. Aucun des sous-délais du DIDO n'a évolué significativement entre les deux périodes (Tableau 1), le sous-délai $\mathrm{D} 2$ a fluctué de 6 min en médiane $(p=0,12)$ avec l'application du REOT. Le DIDO était inférieur ou égal à $30 \mathrm{~min}$ pour aucun patient de la période 1 et pour 2 patients $(6 \%)$ de la période 2 $(p=0,11)$, respectivement de 28 et $30 \mathrm{~min}$.

En périodes 1 et 2 , il y a eu respectivement $8(12 \%)$ et 6 $(18 \%)$ patients thrombolysés $(p=0,39)$. Les délais médians DIDO de ces patients thrombolysés étaient de $75 \mathrm{~min}$ [53-83] en période 1 et de $63 \mathrm{~min}$ [54-78] en période 2 $(p=0,79)$.

Tableau 1 Délais DIDO (door in-door out) et sous-délais (D1, D2, D3, D4) par période

\begin{tabular}{|llll|}
\hline Délais & $\begin{array}{l}\text { Période 1 } \\
\text { 2014-2015 }\end{array}$ & $\begin{array}{l}\text { Période 2 } \\
\mathbf{2 0 1 6}\end{array}$ & Valeur de $\boldsymbol{p}$ \\
\hline D1 & $10[5-30]$ & $8[3-16]$ & 0,18 \\
D2 & $15[8-34]$ & $9[6-26]$ & 0,12 \\
D3 & $31[25-40]$ & $32[27-35]$ & 0,69 \\
D4 & $15[10-20]$ & $18[10-23]$ & 0,38 \\
DIDO & $88[65-135]$ & $72[61-93]$ & 0,06 \\
\hline \multicolumn{4}{l}{ Les délais sont exprimés en minutes avec médiane [interquar- } \\
tile]. Les définitions des sous-délais D1, D2, D3, D4 sont détail- \\
lées en Fig. 1 \\
\hline
\end{tabular}

\section{Discussion}

Les SU sans cardiologie interventionnelle sont confrontés à un double impératif concernant un SCAST + : commencer le traitement antithrombotique en urgence et organiser rapidement le transfert du patient en CCI pour une ACP. Si le délai door to balloon (DTB) est reconnu comme capital dans les recommandations internationales $[1,4,5]$, le délai DIDO est moins souvent évalué.

Dans notre étude, le DIDO a fluctué de 16 min en médiane entre les périodes 1 et 2 . Ce résultat n'est pas significatif $(p=0,06)$, probablement par manque de puissance, et ne permet pas de conclure à une amélioration réelle du délai. Toutefois, l'écart interquartile du DIDO est de $70 \mathrm{~min}$ en période 1 et $32 \mathrm{~min}$ en période 2, ce qui témoigne d'une distribution plus homogène des DIDO au cours du temps avec l'évolution de la procédure SCAST + du RESSIF. Cette optimisation n'est cependant pas suffisante : le DIDO était systématiquement au-delà des recommandations au cours de la période 1 , et seulement 2 patients $(6 \%)$ ont présenté un DIDO inférieur ou égal à $30 \mathrm{~min}$ au cours de la période 2 $(p=0,11)$. L'analyse des sous-délais du DIDO montre que c'est le moment d'appel au SAMU, sous-délai D2, qui semble le plus impacter le DIDO. Le D2 a diminué de 6 min, alors que D1 a diminué de 2 min, D3 et D4 ont augmenté respectivement de 1 et de 3 min entre les deux périodes (Tableau 1). Ce gain de 6 min en médiane est à rapporter au REOT institué début 2016 dans le protocole RESSIF. Ce concept du REOT peut être difficile à concevoir, à savoir prioriser l'appel au SAMU sur un simple ECG, avant tout examen clinique ou décision thérapeutique. Cependant, cette manière de procéder est cohérente, car dans un SU sans CCI, tout SCAST+ est quasi systématiquement transféré. Le délai d'angioplastie étant une priorité, il est logique que toute action qui réduit le DIDO agisse positivement sur le délai DTB. L'activation au plus tôt du SMUR secondaire devient une nécessité, et le REOT un moyen simple que nous avons diffusé au sein du RESSIF. Le temps de route du SMUR secondaire vers le SU (délai D3) est mis à profit pour finaliser l'examen clinique et les traitements, y compris la thrombolyse si justifiée.

Le DIDO a été évalué dans d'autres études. Dans la région du Québec, Lambert et al. rapportent sur une cohorte de 988 patients transférés en CCI un DIDO médian à 51 min [9]. Dans ce travail publié en 2014, le principal sous-délai contribuant à allonger le DIDO est le temps entre l'ECGq et l'activation du transfert en CCI, ce qui correspond dans notre étude au sous-délai D2. Les auteurs concluent que toute intervention visant à faciliter ou accélérer la décision de transfert en CCI avait le meilleur impact sur la diminution du délai de reperfusion coronaire. Ce constat est en accord avec la notion de REOT que nous avons développée. 
Les recommandations précisent que le DIDO devrait être inférieur ou égal à 30 min [4]. Dans les travaux de Lambert et al. [9] et de Wang et al. [6], le DIDO était inférieur ou égal à $30 \mathrm{~min}$ pour respectivement 14 et $11 \%$ des patients. En France, dans le réseau RENAU (Registre nord alpin des urgences), le DIDO des SU sans CCI était mesuré à 91,5 min entre les périodes 2012 et 2014, avec un délai inférieur ou égal à 30 min pour seulement $2 \%$ des patients [10]. Les résultats de ces études montrent que des efforts ciblés sont nécessaires et que des solutions pour atteindre cet objectif sont à explorer. Le REOT peut être un moyen encourageant pour y parvenir.

Le taux de thrombolyse était de $12 \%$ en période 1 et concernait $18 \%$ des SCAST + au SU en 2016. Ce taux peut paraître faible au vu de nos délais DIDO. Le délai douleur thoracique-SU était souvent supérieur à 2 heures chez les patients âgés de plus de 75 ans, ce qui implique, dans notre protocole RESSIF, le choix d'une ACP primaire plutôt qu'une thrombolyse. Toutefois, notre taux de thrombolyse est supérieur au $6 \%$ rapporté dans un registre national français [3]. Dans une étude multicentrique aux États-Unis publiée en 2011, Herrin et al. constataient que le DIDO excédait 90 min pour $31 \%$ des SCAST+ et que le délai DTB dépassait finalement les seuils recommandés. Selon les auteurs, un nombre significatif de patients auraient dû être thrombolysés [11]. Ainsi, dans les SU sans CCI, la thrombolyse est probablement sous-utilisée, et la mesure du DIDO pourrait être un élément déterminant pour décider entre ACP primaire et thrombolyse préalable.

Notre démarche régionale du RESSIF n'a été possible que par la création d'un réseau transversal de soins. Tous les acteurs de l'urgence coronaire ont coopéré ensemble pour fluidifier la prise en charge des SCAST+ en SMUR, au SU et en CCI. En 2006, Bradley et al. [12] montraient que les hôpitaux qui avaient les meilleurs délais de prise en charge étaient ceux qui coopéraient au sein d'un réseau, avec des protocoles partagés et une restitution périodique des données de prise en charge afin de motiver les équipes et de renforcer l'adhésion aux protocoles $[12,13]$. La création de réseaux de soins transversaux est ainsi impérative $[4,5]$.

Notre étude a plusieurs limites. Nous n'avons pas mis en évidence de modification significative du DIDO, ce qui limite l'interprétation des résultats. Cela est probablement lié à un manque de puissance dû aux faibles effectifs de patients inclus. Il faut rappeler que le RESSIF n'a été créé qu'en 2013, et nous rapportons ici les résultats limités à deux SU pilotes. Nous prévoyons de mesurer le DIDO et l'impact du REOT dans l'ensemble du réseau, afin d'obtenir des données à plus grande échelle pour évaluer la validité interne et la pertinence clinique de notre démarche. Notre étude ne concerne que notre région géographique, avec ses spécificités de population et de moyens logistiques. La généralisation à d'autres systèmes de soins pourrait ne pas s'appliquer. Néanmoins, les mesures établies pour faire évoluer le DIDO au sein du RESSIF et les procédures communes qui en découlent pourraient être envisagées dans des régions non encore organisées en réseau. D'autres réseaux français existent déjà et s'inscrivent dans une démarche régionale d'amélioration de prise en charge du SCA [10]. Enfin, nous n'avons pas évalué dans notre étude l'impact du DIDO sur la morbimortalité. Le faible effectif de notre population d'étude ne nous permet pas ici de mesurer cette association. Le recueil continu des SCAST $+\mathrm{du}$ RESSIF permettrait un plus grand nombre d'inclusions pour évaluer la corrélation entre DIDO et morbimortalité, qui a par ailleurs été affirmée dans d'autres études [6].

\section{Conclusion}

Le délai DIDO est un indicateur de qualité indispensable à mesurer dans les SU sans CCI pour optimiser le délai d'ACP. Si un DIDO inférieur ou égal à $30 \mathrm{~min}$ semble difficile à atteindre, le REOT pourra être un moyen pour tendre vers cet objectif recommandé, et sa faisabilité dans les SU mériterait d'être évaluée plus largement. La mise en place de réseaux de soins impliquant les SAMU, SMUR, SU et CCI est un préalable indispensable pour établir des protocoles et procédures partagés. Le pronostic des SCAST+ est conditionné en majeure partie par cette démarche.

Conflits d'intérêts : les auteurs déclarents ne pas avoir de liens d'intérêts.

\section{Références}

1. Puymirat E, Caudron J, Steg PG, et al (2017) Prognostic impact of non-compliance with guidelines-recommended times to reperfusion therapy in ST-elevation myocardial infarction. The FASTMI 2010 registry. Eur Heart J Acute Cardiovasc Care 6:26-33

2. Puymirat E, Simon T, Steg PG, et al (2012) Association of changes in clinical characteristics and management with improvement in survival among patients with ST-elevation myocardial infarction. JAMA 308:998-1006

3. Belle L, Cayla G, Cottin Y, et al (2017) French registry on acute ST-elevation and non-ST-elevation myocardial infarction 2015 (FAST-MI 2015). Design and baseline data. Arch Cardiovasc Dis 110:366-78

4. O'Gara PT, Kushner FG, Ascheim DD, et al (2013) ACCF/AHA guideline for the management of ST-elevation myocardial infarction: executive summary a report of the American College of Cardiology Foundation/American Heart Association task force on practice guidelines. J Am Coll Cardiol 61:485-510

5. Windecker S, Kolh P, Alfonso F, et al (2014) 2014 ESC/EACTS guidelines on myocardial revascularization: the Task Force on myocardial revascularization of the European Society of Cardiology (ESC) and the European Association for Cardio-Thoracic Surgery (EACTS) developed with the special contribution of the European Association of Percutaneous Cardiovascular Interventions (EAPCI). EHJ 35:2541-619 
6. Wang TY, Nallamothu BK, Krumholz HM, et al (2011) Association of door-in to door-out time with reperfusion delays and outcomes among patients transferred for primary percutaneous coronary intervention. JAMA 305:2540-7

7. Lapandry C, Laperche T, Lambert Y, et al (2005) Pre-hospital management of acute coronary syndromes with ST-elevation in the Ile de France region: the E-must registry. Arch Mal Coeur Vaiss 98:1137-42

8. Ward MJ, Kripalani S, Storrow AB, et al (2015) Timeliness of inter-facility transfer for emergency department patients with ST-elevation myocardial infarction. Am J Emerg Med 33:423-9

9. Lambert LJ, Brown KA, Boothroyd LJ, et al (2014) Transfer of patients with ST-elevation myocardial infarction for primary percutaneous coronary intervention: a province-wide evaluation of "doorin to door-out" delays at the first hospital. Circulation 129:2653-60
10. Clot S, Rocher T, Morvan C, et al (2016) Door in - door out assessment of patients admitted with acute ST-segment elevation myocardial infarction in hospitals without catheterization facilities. Ann Cardiol Angeiol 65:375

11. Herrin J, Miller LE, Turkmani DF, et al (2011) National performance on door-in to door-out time among patients transferred for primary percutaneous coronary intervention. Arch Intern Med 171:1879-86

12. Bradley EH, Curry LA, Webster TR, et al (2006) Achieving rapid door-to balloon times: how top hospitals improve complex clinical systems. Circulation 113:1079-85

13. Bradley EH, Herrin J, Wang Y, et al (2006) Strategies for reducing the door-to-balloon time in acute myocardial infarction. N Engl J Med 355:2308-20 